\title{
TRI-QUOTIENT MAPS ARE PRESERVED BY INFINITE PRODUCTS
}

\author{
V. V. USPENSKIJ \\ (Communicated by Franklin D. Tall)
}

\begin{abstract}
The theorem stated in the title answers a question of E. Michael
\end{abstract} (1977).

\section{INTRODUCTION}

By the product of a family of maps $f_{\alpha}: X_{\alpha} \rightarrow Y_{\alpha}, \alpha \in A$, we mean the map $\prod_{\alpha \in A} f_{\alpha}: \prod_{\alpha \in A} X_{\alpha} \rightarrow \prod_{\alpha \in A} Y_{\alpha}$ defined by $\prod_{\alpha \in A} f_{\alpha}\left(\left\{x_{\alpha}\right\}\right)=\left\{f_{\alpha}\left(x_{\alpha}\right)\right\}$. All maps are assumed to be continuous.

The product of any family of perfect maps is perfect [E, Theorem 3.7.9]. This theorem is a generalization of the Tikhonov theorem on products of compact spaces, since a space $X$ is compact if and only if the constant map of $X$ to a singleton is perfect. It is natural to ask what other classes of maps are preserved by products. For instance, the class of open maps has this property, and so does the class of bi-quotient maps [M1], while the classes of closed or quotient maps do not. Moreover, the product $f \times \mathrm{id}_{Z}$ is closed for every space $Z$ if and only if $f$ is perfect [E, Theorem 3.7.14]. Similarly, $f \times \mathrm{id}_{Z}$ is quotient for every $Z$ if and only if $f$ is bi-quotient [H], [M1].

Recall that an onto map $f: X \rightarrow Y$ is bi-quotient [M1] if for any $y \in Y$ and any cover $\mathscr{W}$ of $f^{-1}(y)$ by open subsets of $X$ there exists a finite subfamily $\mathscr{E} \subset \mathscr{W}$ such that $y$ belongs to the interior of $f(\bigcup \mathscr{E})$. The following subclass of the class of bi-quotient maps was defined by E. Michael in [M2]:

Definition 1. An onto map $f: X \rightarrow Y$ is tri-quotient if one can assign to each open $U \subset X$ an open $U^{*} \subset Y$ such that:

(1) $U^{*} \subset f(U)$;

(2) $X^{*}=Y$;

(3) $U \subset V$ implies $U^{*} \subset V^{*}$;

(4) if $y \in U^{*}$ and if $\mathscr{W}$ is a cover of $f^{-1}(y) \cap U$ by open subsets of $X$, then $y \in(\bigcup \mathscr{E})^{*}$ for some finite $\mathscr{E} \subset \mathscr{W}$.

A mapping $U \mapsto U^{*}$ satisfying (1)-(4) will be called t-assignment.

Open onto maps and perfect onto maps are tri-quotient [M2]. Inductively tri-quotient maps are tri-quotient [M2]. (A map $f: X \rightarrow Y$ is inductively $\mathscr{P}$,

Received by the editors October 6, 1993 and, in revised form, April 25, 1994.

1991 Mathematics Subject Classification. Primary 54C10.

Key words and phrases. Tikhonov theorem, perfect map, bi-quotient map, tri-quotient map.

Research supported by a Humboldt Research Fellowship. 
where $\mathscr{P}$ is a property of maps, if there exists $X^{\prime} \subset X$ such that $f\left(X^{\prime}\right)=$ $Y$ and the restriction $f \mid X^{\prime}: X^{\prime} \rightarrow Y$ has $\mathscr{P}$.) In particular, inductively perfect maps are tri-quotient. For some spaces, the converse is also true. For example, any tri-quotient map of a regular sieve-complete (= monotonically Čech-complete [CČN]) space onto a paracompact space is inductively perfect [M2], [JW] (for open maps of Čech-complete spaces this is due to Pasynkov [P]). For such spaces, tri-quotient maps coincide with inductively perfect maps and hence are preserved by products. E. Michael asked [M2] whether this is true in general: are tri-quotient maps preserved by arbitrary products? The question was repeated in an early version of [M3]. E. Michael proved [M2, Theorem 7.2] that the answer is positive for finite products. The aim of this paper is to show that this is also true for infinite products:

Theorem 1. Let $f_{\alpha}: X_{\alpha} \rightarrow Y_{\alpha}, \alpha \in A$, be a family of tri-quotient maps. Then the product $\prod_{\alpha \in A} f_{\alpha}: \prod_{\alpha \in A} X_{\alpha} \rightarrow \prod_{\alpha \in A} Y_{\alpha}$ is tri-quotient.

We first describe the idea of the proof. For every topological space $X$ we define a non-Hausdorff topological space $\mathfrak{Q}(X)$ (Definition 3), consisting of systems of open sets in $X$ with some special properties. The definition of tri-quotient maps has a natural reformulation in terms of such spaces (Proposition 1). We show in Section 2 that Theorem 1 follows from Theorem 2, which asserts that for any family $\left\{X_{\alpha}: \alpha \in A\right\}$ of topological spaces there exists a map from $\prod_{\alpha \in A} \mathfrak{Q}\left(X_{\alpha}\right)$ to $\mathfrak{Q}\left(\prod_{\alpha \in A} X_{\alpha}\right)$ which preserves supports (Definitions 4 and 5). Theorem 2 is a consequence of Theorem 3, which describes such a map explicitly. Theorem 3 is proved in Section 3, and Section 4 contains some comments and remarks.

\section{REDUCTION TO THEOREM 2}

The collection of open subsets of a topological space $X$ will be denoted by $\mathscr{T}(X)$. We start with a reformulation of the definition of tri-quotient maps (Proposition 1).

Definition 2. Let $X$ be a topological space. A system $\mathscr{S} \subset \mathscr{T}(X)$ of open sets in $X$ is a $Q$-system if the following three conditions hold:

(1) if $\mathscr{E} \subset \mathscr{T}(X)$ and $\bigcup \mathscr{E} \in \mathscr{S}$, then $\bigcup \mathscr{F} \in \mathscr{S}$ for some finite $\mathscr{F} \subset \mathscr{E}$;

(2) if $U \in \mathscr{S}, V \in \mathscr{T}(X)$ and $U \subset V$, then $V \in \mathscr{S}$;

(3) $X \in \mathscr{S}$ and $\varnothing \notin \mathscr{S}$.

If $\mathscr{B}$ is a base for $X$, then it suffices if condition (1) is satisfied for all $\mathscr{E} \subset \mathscr{B}$.

Definition 3. For a topological space $X$, we denote by $\mathfrak{Q}(X)$ the space of all $Q$-systems on $X$, topologized as follows:

For $U \in \mathscr{T}(X)$, let $\tilde{U}=\{\mathscr{S} \in \mathfrak{Q}(X): U \in \mathscr{S}\}$. The topology of $\mathfrak{Q}(X)$ is generated by the family $\{\tilde{U}: U \in \mathscr{T}(X)\}$.

The space $\mathfrak{Q}(X)$ is $T_{0}$ but usually not $T_{1}$. There is a natural continuous map $i: X \rightarrow \mathfrak{Q}(X)$ defined by $i(x)=\{U \in \mathscr{T}(X): x \in U\}$. If $X$ is $T_{0}$, this map is a homeomorphic embedding.

Definition 4. A $Q$-system $\mathscr{S}$ on $X$ is supported by a subset $E \subset X$ if the following condition holds: if $U, V \in \mathscr{T}(X), U \cap E=V \cap E$ and $U \in \mathscr{S}$, then $V \in \mathscr{S}$. 
Note that if $\mathscr{S} \in \mathfrak{Q}(X)$ is supported by $E \subset X$, then every element of $\mathscr{S}$ meets $E$.

Proposition 1. A map $f: X \rightarrow Y$ is tri-quotient if and only if there exists a (continuous) map $r: Y \rightarrow \mathfrak{Q}(X)$ such that for every $y \in Y$ the $Q$-system $r(y)$ is supported by $f^{-1}(y)$.

This is essentially the same characterization of tri-quotient maps as the one given in [O].

Proof. Given a $t$-assignment $U \mapsto U^{*}\left(U \in \mathscr{T}(X), U^{*} \in \mathscr{T}(Y)\right)$, define $r(y)=\left\{U \in \mathscr{T}(X): y \in U^{*}\right\}$. Conversely, given $r$, define a $t$-assignment by $U^{*}=\{y \in Y: U \in r(y)\}$.

Definition 5. Let $\left\{X_{\alpha}: \alpha \in A\right\}$ be a family of topological spaces. We say that a map $\boldsymbol{\Theta}: \prod_{\alpha \in A} \mathfrak{Q}\left(X_{\alpha}\right) \rightarrow \mathfrak{Q}\left(\prod_{\alpha \in A} X_{\alpha}\right)$ is support-preserving if the following condition holds: Suppose that $\mathscr{S}_{\alpha} \in \mathfrak{Q}\left(X_{\alpha}\right)$ and $E_{\alpha} \subset X_{\alpha}$ for every $\alpha \in A$. If each $\mathscr{S}_{\alpha}$ is supported by $E_{\alpha}$, then $\Theta\left(\left\{\mathscr{S}_{\alpha}\right\}\right)$ is supported by $\prod_{\alpha \in A} E_{\alpha}$.

Theorem 2. For any family $\left\{X_{\alpha}: \alpha \in A\right\}$ of topological spaces there exists a support-preserving continuous map $\Theta: \prod_{\alpha \in A} \mathfrak{Q}\left(X_{\alpha}\right) \rightarrow \mathfrak{Q}\left(\prod_{\alpha \in A} X_{\alpha}\right)$.

We show that our main result, Theorem 1, follows from Theorem 2 . Let $f_{\alpha}: X_{\alpha} \rightarrow Y_{\alpha}, \alpha \in A$, be a family of tri-quotient maps. In virtue of Proposition 1 , for every $\alpha \in A$ there exists a map $r_{\alpha}: Y_{\alpha} \rightarrow \mathfrak{Q}\left(X_{\alpha}\right)$ such that $r_{\alpha}(y)$ is supported by $f_{\alpha}^{-1}(y)$ for every $y \in Y_{\alpha}$. Define $r: \prod_{\alpha} Y_{\alpha} \rightarrow \mathfrak{Q}\left(\prod_{\alpha} X_{\alpha}\right)$ as the composition of $\prod_{\alpha} r_{\alpha}: \prod_{\alpha} Y_{\alpha} \rightarrow \prod_{\alpha} \mathfrak{Q}\left(X_{\alpha}\right)$ and a map $\Theta: \prod_{\alpha} \mathfrak{Q}\left(X_{\alpha}\right) \rightarrow$ $\mathfrak{Q}\left(\prod_{\alpha} X_{\alpha}\right)$ such as in Theorem 2 . Since $\Theta$ preserves supports, for every $y=$ $\left\{y_{\alpha}: \alpha \in A\right\}$ the $Q$-system $r(y)$ is supported by $\prod_{\alpha} f_{\alpha}^{-1}\left(y_{\alpha}\right)=\left(\prod_{\alpha} f_{\alpha}\right)^{-1}(y)$. Another application of Proposition 1 shows that $\prod_{\alpha} f_{\alpha}$ is tri-quotient.

\section{Proofs}

Theorem 3 below explicitly describes a map with the properties required by Theorem 2.

Definition 6. Given a $Q$-system $\mathscr{S} \in \mathfrak{Q}(X)$, let us say that a closed set $F \subset X$ is $\mathscr{S}$-big if $F$ meets every element of $\mathscr{S}$. (Note that, by condition (2) of Definition 2, $F$ is $\mathscr{S}$-big if and only if $X \backslash F \notin \mathscr{S}$.) If $\left\{X_{\alpha}: \alpha \in A\right\}$ is a family of topological spaces, $\mathscr{S}_{\alpha} \in \mathfrak{Q}\left(X_{\alpha}\right)$ and $F_{\alpha}$ is an $\mathscr{S}_{\alpha}$-big closed subset of $X_{\alpha}$ for every $\alpha \in A$, we say that the product $\prod_{\alpha \in A} F_{\alpha}$ is an $\left\{\mathscr{S}_{\alpha}\right\}$-big box in $\prod_{\alpha \in A} X_{\alpha}$.

Theorem 3. Let $\left\{X_{\alpha}: \alpha \in A\right\}$ be a family of topological spaces, $\mathscr{S}_{\alpha} \in \mathfrak{Q}\left(X_{\alpha}\right)$ for every $\alpha \in A$. Let $\boldsymbol{\Theta}\left(\left\{\mathscr{S}_{\alpha}\right\}\right)$ be the family of all open sets $U$ in $X=\prod_{\alpha \in A} X_{\alpha}$ with the following property: $U$ meets every $\left\{\mathscr{S}_{\alpha}\right\}$-big box in $X$. Then $\boldsymbol{\Theta}\left(\left\{\mathscr{S}_{\alpha}\right\}\right)$ is a $Q$-system on $X$, and the function $\Theta: \prod_{\alpha \in A} \mathfrak{Q}\left(X_{\alpha}\right) \rightarrow \mathfrak{Q}(X)$ is continuous and support-preserving.

Proof. Let $\mathscr{S}=\boldsymbol{\Theta}\left(\left\{\mathscr{S}_{\alpha}\right\}\right)$. Our proof proceeds in three parts.

(a) We show that $\mathscr{S}$ is a $Q$-system. In virtue of the remark after Definition 2, it suffices to prove the following: if $\mathscr{E}=\{V(\gamma): \gamma \in \Gamma\}$ is a family of basic open sets in $X$ and $\bigcup \mathscr{F} \notin \mathscr{S}$ for every finite $\mathscr{F} \subset \mathscr{E}$, then $\bigcup \mathscr{E} \notin \mathscr{S}$. The assumption that $V(\gamma)$ is basic means that $V(\gamma)=\prod_{\alpha \in A} V_{\alpha}(\gamma)$, where 
$V_{\alpha}(\gamma) \in \mathscr{T}\left(X_{\alpha}\right)$ and $V_{\alpha}(\gamma) \neq X_{\alpha}$ only for $\alpha \in A_{\gamma}$ for some finite subset $A_{\gamma}$ of $A$.

Lemma 1. The union of the family $\mathscr{E}=\{V(\gamma): \gamma \in \Gamma\}$ of basic open subsets $V(\gamma)=\prod_{\alpha \in A} V_{\alpha}(\gamma)$ of $X$ is not in $\mathscr{S}$ if and only if there exists a function $\phi: \Gamma \rightarrow A$ such that for every $\alpha \in A$ the set $U_{\alpha}=\bigcup\left\{V_{\alpha}(\gamma): \gamma \in \Gamma, \phi(\gamma)=\alpha\right\}$ is not in $\mathscr{S}_{\alpha}$.

Proof. If $\phi$ is such a function, let $F_{\alpha}=X_{\alpha} \backslash U_{\alpha}$. Since $U_{\alpha} \notin \mathscr{S}_{\alpha}$, the set $F_{\alpha}$ is $\mathscr{S}_{\alpha}$-big. Hence $F=\prod_{\alpha} F_{\alpha}$ is an $\left\{\mathscr{S}_{\alpha}\right\}$-big box in $X$. For every $\gamma \in \Gamma$, the set $V(\gamma)$ misses $F$, since $V_{\alpha}(\gamma)$ misses $F_{\alpha}$ for $\alpha=\phi(\gamma)$. It follows that $\bigcup \mathscr{E}$ misses $F$, which means that $\bigcup \mathscr{E} \notin \mathscr{S}$.

Conversely, suppose that $\bigcup \mathscr{E} \notin \mathscr{S}$. Then $\bigcup \mathscr{E}$ misses some $\left\{\mathscr{S}_{\alpha}\right\}$-big box $F=\prod_{\alpha} F_{\alpha}$. For every $\gamma \in \Gamma$ there exists an $\alpha \in A_{\gamma}$ such that $V_{\alpha}(\gamma)$ misses $F_{\alpha}$. Put $\alpha=\phi(\gamma)$. Then $U_{\alpha}=\bigcup\left\{V_{\alpha}(\gamma): \gamma \in \Gamma, \phi(\gamma)=\alpha\right\} \subset X_{\alpha} \backslash F_{\alpha}$, so $U_{\alpha}$ is not in $\mathscr{S}_{\alpha}$ and $\phi$ is as required.

Since for every finite $\mathscr{F} \subset \mathscr{E}$ the set $\bigcup \mathscr{F}$ is not in $\mathscr{S}$, Lemma 1 applied to $\mathscr{F}$ instead of $\mathscr{E}$ yields for every finite $K \subset \Gamma$ a function $\phi_{K}: K \rightarrow A$ such that

$$
\bigcup\left\{V_{\alpha}(\gamma): \gamma \in K, \phi_{K}(\gamma)=\alpha\right\} \notin \mathscr{S}_{\alpha}
$$

for every $\alpha \in A$. If $\phi$ is as in Lemma 1 , then $\phi(\gamma) \in A_{\gamma}$ for every $\gamma \in \Gamma$, so $\phi \in \prod_{\gamma \in \Gamma} A_{\gamma}$. Similarly, $\phi_{K} \in \prod_{\gamma \in K} A_{\gamma}$ for every finite $K \subset \Gamma$.

Lemma 2. Let $\left\{A_{\gamma}: \gamma \in \Gamma\right\}$ be a family of finite sets, and let $\phi_{K} \in \prod_{\gamma \in K} A_{\gamma}$ for every finite $K \subset \Gamma$. Then there exists $\phi \in \prod_{\gamma \in \Gamma} A_{\gamma}$ with the following property: For any finite $L \subset \Gamma$ there exists a finite $K \subset \Gamma$ such that $L \subset K$ and $\phi\left|L=\phi_{K}\right| L$.

Proof. For each finite $K \subset \Gamma$ pick an extension $\psi_{K} \in \prod_{\gamma \in \Gamma} A_{\gamma}$ of $\phi_{K}$. Consider the net $s=\left\{\psi_{K}: K \subset \Gamma, K\right.$ finite $\}$, where the index set is ordered by inclusion. By the Tikhonov theorem, the product space $P=\prod_{\gamma \in \Gamma} A_{\gamma}$, where each of the finite sets $A_{\gamma}$ carries the discrete topology, is compact. Hence the net $s$ has a cluster point $\phi$ in $P$. Let us check that $\phi$ is as required. Suppose $L$ is a finite subset of $\Gamma$. Let $O$ be the neighborhood of $\phi$ in $P$ consisting of all $\psi \in P$ with $\psi|L=\phi| L$. Since $\phi$ is a cluster point of $s$, there is a finite set $K \subset \Gamma$ such that $L \subset K$ and $\psi_{K} \in O$. Then $\phi\left|L=\psi_{K}\right| L=\phi_{K} \mid L$.

Lemma 2 applied to the family $\left\{\phi_{K}\right\}$ constructed above yields a function $\phi: \Gamma \rightarrow A$. Let $\mathscr{E}_{\alpha}=\left\{V_{\alpha}(\gamma): \gamma \in \Gamma, \phi(\gamma)=\alpha\right\}$ for $\alpha \in A$. In virtue of Lemma 1 , to prove that $\bigcup \mathscr{E} \notin \mathscr{S}$ it suffices to show that $\bigcup \mathscr{E}_{\alpha} \notin \mathscr{S}_{\alpha}$ for every $\alpha \in A$. Let $\mathscr{F}_{\alpha}=\left\{V_{\alpha}(\gamma): \gamma \in L, \phi(\gamma)=\alpha\right\}$ be any finite subfamily of $\mathscr{E}_{\alpha}$. Applying the condition of Lemma 2, choose a finite subset $K \subset \Gamma$ with $L \subset K$ and $\phi\left|L=\phi_{K}\right| L$. Then $\bigcup \mathscr{F}_{\alpha} \subset \bigcup\left\{V_{\alpha}(\gamma): \gamma \in K, \phi_{K}(\gamma)=\alpha\right\}$. By the choice of $\phi_{K}$, the last union is not in $\mathscr{S}_{\alpha}$, hence also $\bigcup \mathscr{F}_{\alpha} \notin \mathscr{S}_{\alpha}$. Since this holds for every finite subfamily of $\mathscr{E}_{\alpha}$ and since $\mathscr{S}_{\alpha}$ is a $Q$-system, it follows that $\bigcup \mathscr{E}_{\alpha} \notin \mathscr{S}_{\alpha}$. As we have noted, this proves that $\bigcup \mathscr{E} \notin \mathscr{S}$. Thus $\mathscr{S}$ is a $Q$-system.

(b) We now verify that $\Theta: \prod_{\alpha} \mathfrak{Q}\left(X_{\alpha}\right) \rightarrow \mathfrak{Q}(X)$ is continuous at $\left\{\mathscr{S}_{\alpha}\right\}$. Let $U \in \mathscr{S}$. We must show that there is a basic neighborhood $\prod_{\alpha} \mathfrak{W}_{\alpha}$ of $\left\{\mathscr{S}_{\alpha}\right\}$ in 
$\prod_{\alpha} \mathfrak{Q}\left(X_{\alpha}\right)$ such that $\boldsymbol{\Theta}\left(\prod_{\alpha} \mathfrak{W}_{\alpha}\right) \subset \tilde{U}$ (notation of Definition 3). Since $\mathscr{S}$ is a $Q$-system, there exists a finite family $\{V(\gamma): \gamma \in \Gamma\}$ of basic open sets such that $V=\bigcup\{V(\gamma): \gamma \in \Gamma\} \subset U$ and $V \in \mathscr{S}$. Write $V(\gamma)$ as $V(\gamma)=\prod_{\alpha \in A} V_{\alpha}(\gamma)$, and for each $\alpha$ let $\mathscr{P}_{\alpha}$ be the finite set of all elements of $\mathscr{S}_{\alpha}$ which can be represented as the union of some subfamily of $\left\{V_{\alpha}(\gamma): \gamma \in \Gamma\right\}$. Lemma 1 implies that for any function $\phi: \Gamma \rightarrow A$ there exists $\alpha \in A$ such that the union of the family $\left\{V_{\alpha}(\gamma): \gamma \in \Gamma, \phi(\gamma)=\alpha\right\}$ is in $\mathscr{S}_{\alpha}$ and hence also in $\mathscr{P}_{\alpha}$. Suppose that $\mathscr{S}_{\alpha}^{\prime} \in \mathfrak{Q}\left(X_{\alpha}\right)$ and $\mathscr{P}_{\alpha} \subset \mathscr{S}_{\alpha}^{\prime}$ for every $\alpha \in A$. For any $\phi: \Gamma \rightarrow A$ there exists $\alpha \in A$ such that the union of the family $\left\{V_{\alpha}(\gamma): \gamma \in \Gamma, \phi(\gamma)=\alpha\right\}$ is in $\mathscr{S}_{\alpha}^{\prime}$. Applying Lemma 1 again, we see that $V \in \Theta\left(\left\{\mathscr{S}_{\alpha}^{\prime}\right\}\right)$ and hence also $U \in \Theta\left(\left\{\mathscr{S}_{\alpha}^{\prime}\right\}\right)$. In other words, if $\mathfrak{W}_{\alpha}$ is the neighborhood of $\mathscr{S}_{\alpha}$ in $\mathfrak{Q}\left(X_{\alpha}\right)$ consisting of all $Q$-systems $\mathscr{S}_{\alpha}^{\prime}$ with $\mathscr{P}_{\alpha} \subset \mathscr{S}_{\alpha}^{\prime}$, then $\Theta$ maps the product $\prod_{\alpha} \mathfrak{W}_{\alpha}$ into the neighborhood $\tilde{U}$ of $\mathscr{S}$ in the space $\mathfrak{Q}(X)$. Since $\mathscr{P}_{\alpha}=\left\{X_{\alpha}\right\}$ and hence $\mathfrak{W}_{\alpha}=\mathfrak{Q}\left(X_{\alpha}\right)$ for all but finitely many indices $\alpha \in A$, the product $\prod_{\alpha} \mathfrak{W}_{\alpha}$ is a neighborhood of $\left\{\mathscr{S}_{\alpha}\right\}$ in $\prod_{\alpha} \mathfrak{Q}\left(X_{\alpha}\right)$. Thus $\Theta$ is continuous.

(c) It remains to show that $\Theta$ is support-preserving. For a map $f: Y \rightarrow Z$, define a map $\mathfrak{Q} f: \mathfrak{Q}(Y) \rightarrow \mathfrak{Q}(Z)$ by $\mathfrak{Q} f(\mathscr{S})=\left\{U \in \mathscr{T}(Z): f^{-1}(U) \in \mathscr{S}\right\}$. It is easy to see that $\mathfrak{Q} f(\mathscr{S})$ is a $Q$-system on $Z$ and that $\mathfrak{Q} f$ is continuous. If $Y \subset Z$ and $j: Y \rightarrow Z$ is the inclusion, the set of all $Q$-systems on $Z$ supported by $Y$ coincides with the range of the map $\mathfrak{Q} j: \mathfrak{Q}(Y) \rightarrow \mathfrak{Q}(Z)$.

Suppose $\mathscr{S}_{\alpha} \in \mathfrak{Q}\left(X_{\alpha}\right)$ is supported by $E_{\alpha} \subset X_{\alpha}$ for every $\alpha \in A$. This means that every $\mathscr{S}_{\alpha}$ is in the range of the map $\mathfrak{Q} i_{\alpha}: \mathfrak{Q}\left(E_{\alpha}\right) \rightarrow \mathfrak{Q}\left(X_{\alpha}\right)$, where $i_{\alpha}$ denotes the inclusion of $E_{\alpha}$ into $X_{\alpha}$. We have to show that $\Theta\left(\left\{\mathscr{S}_{\alpha}\right\}\right)$ is supported by $\prod_{\alpha} E_{\alpha}$ or, equivalently, is in the range of $\mathfrak{Q}\left(\prod_{\alpha} i_{\alpha}\right): \mathfrak{Q}\left(\prod_{\alpha} E_{\alpha}\right) \rightarrow$ $\mathfrak{Q}\left(\prod_{\alpha} X_{\alpha}\right)$. This follows from a more general fact:

Lemma 3. Let $\left\{f_{\alpha}: \alpha \in A\right\}$ be a family of maps, $f_{\alpha}: Y_{\alpha} \rightarrow X_{\alpha}$. Let $\boldsymbol{\Theta}_{X}$ : $\prod_{\alpha} \mathfrak{Q}\left(X_{\alpha}\right) \rightarrow \mathfrak{Q}\left(\prod_{\alpha} X_{\alpha}\right)$ and $\Theta_{Y}: \prod_{\alpha} \mathfrak{Q}\left(Y_{\alpha}\right) \rightarrow \mathfrak{Q}\left(\prod_{\alpha} Y_{\alpha}\right)$ be the maps defined in Theorem 3. Then the diagram

$$
\begin{array}{cc}
\prod_{\alpha} \mathfrak{Q}\left(Y_{\alpha}\right) \stackrel{\boldsymbol{\Theta}_{Y}}{\longrightarrow} & \mathfrak{Q}\left(\prod_{\alpha} Y_{\alpha}\right) \\
\prod_{\alpha} \mathfrak{Q} f_{\alpha} \downarrow & \\
\prod_{\alpha} \mathfrak{Q}\left(X_{\alpha}\right) & \underset{\mathfrak{Q}\left(\prod_{\alpha} f_{\alpha}\right)}{\boldsymbol{\Theta}_{X}} \mathfrak{Q}\left(\prod_{\alpha} X_{\alpha}\right)
\end{array}
$$

commutes.

Proof. Let $\mathscr{P}_{\alpha} \in \mathfrak{Q}\left(Y_{\alpha}\right)$ for every $\alpha \in A$. Let $\mathscr{S} \in \mathfrak{Q}\left(\prod_{\alpha} X_{\alpha}\right)$ and $\mathscr{S}^{\prime} \in$ $\mathfrak{Q}\left(\prod_{\alpha} X_{\alpha}\right)$ be the values at $\left\{\mathscr{P}_{\alpha}\right\}$ of the maps $\mathfrak{Q}\left(\prod_{\alpha} f_{\alpha}\right) \circ \boldsymbol{\Theta}_{Y}$ and $\boldsymbol{\Theta}_{X} \circ \prod_{\alpha} \mathfrak{Q} f_{\alpha}$, respectively. We must show that $\mathscr{S}=\mathscr{S}^{\prime}$.

Let $\mathscr{V}=\left\{\prod_{\alpha \in A} V_{\alpha}(\gamma): \gamma \in \Gamma\right\}$ be a family of basic open sets in $\prod_{\alpha} X_{\alpha}$. For every $\gamma \in \Gamma$ let $W(\gamma)=\prod_{\alpha \in A} f_{\alpha}^{-1}\left(V_{\alpha}(\gamma)\right) \in \mathscr{T}\left(\prod_{\alpha} Y_{\alpha}\right)$, and let $\mathscr{W}=\{W(\gamma)$ : $\gamma \in \Gamma\}$. The following conditions are equivalent:

(1) $\cup \mathscr{V} \in \mathscr{S}$.

(2) $\bigcup \mathscr{W} \in \Theta_{Y}\left(\left\{\mathscr{P}_{\alpha}\right\}\right)$.

(3) For any function $\phi: \Gamma \rightarrow A$ there exists $\alpha \in A$ such that the union of the family $\left\{f_{\alpha}^{-1}\left(V_{\alpha}(\gamma)\right): \gamma \in \Gamma, \phi(\gamma)=\alpha\right\}$ is in $\mathscr{P}_{\alpha}$.

(4) For any function $\phi: \Gamma \rightarrow A$ there exists $\alpha \in A$ such that the union of the family $\left\{V_{\alpha}(\gamma): \gamma \in \Gamma, \phi(\gamma)=\alpha\right\}$ is in $\mathfrak{Q} f_{\alpha}\left(\mathscr{P}_{\alpha}\right)$.

(5) $\bigcup \mathscr{V} \in \mathscr{S}^{\prime}$. 
The equivalences (1) $\Leftrightarrow(2)$ and (3) $\Leftrightarrow$ (4) follow from the definition of $\mathfrak{Q}\left(\prod_{\alpha} f_{\alpha}\right)$ and $\mathfrak{Q} f_{\alpha}$, while the equivalences $(2) \Leftrightarrow(3)$ and $(4) \Leftrightarrow(5)$ follow from Lemma 1. The equivalence of conditions (1) and (5) means that $\mathscr{S}=\mathscr{S}^{\prime}$.

This completes the proof of Theorem 3 and thus also of Theorems 1 and 2 .

\section{REMARKS}

1. Our proof of Theorem 1 does not depend on whether the given family of tri-quotient maps is finite or infinite. As noted in the Introduction, the preservation of tri-quotient maps under finite products was proved by $E$. Michael in [M2]. His proof, rewritten in terms of $Q$-systems, looks as follows.

It suffices to prove Theorem 2 for two factors, that is, to define a continuous support-preserving map $\Theta: \mathfrak{Q}(X) \times \mathfrak{Q}(Y) \rightarrow \mathfrak{Q}(X \times Y)$. Let $\mathscr{S}_{1} \in \mathfrak{Q}(X)$, $\mathscr{S}_{2} \in \mathfrak{Q}(Y)$. For a set $G \subset X \times Y$ and $x \in X$, denote by $G_{x}$ the set of all $y \in Y$ such that $(x, y) \in G$. Define $\Theta\left(\mathscr{S}_{1}, \mathscr{S}_{2}\right)$ to be the family of all open sets $G \subset X \times Y$ with the following property: The set $\left\{x \in X: G_{x} \in \mathscr{S}_{2}\right\}$ is in $\mathscr{S}_{1}$. It is easy to check that $\Theta$ is as required. The definition of $\Theta$ has an equivalent reformulation: an open set $G \subset X \times Y$ is in $\Theta\left(\mathscr{S}_{1}, \mathscr{S}_{2}\right)$ if and only if $G$ contains a finite union $\bigcup_{i=1}^{n} U_{i} \times V_{i}$ of basic open sets such that $\bigcup_{i=1}^{n} U_{i} \in \mathscr{S}_{1}$ and $V_{i} \in \mathscr{S}_{2}, i=1, \ldots, n$.

2 . The preceding remark shows that a map $\Theta$ such as in Theorem 2 is not unique. Consider again the case of two factors $X$ and $Y$. Let $\Theta$ be as in Theorem 3, and let $\Theta^{\prime}$ be as in Remark 1. A union $\bigcup_{i \in I} U_{i} \times V_{i}$ of basic open sets is in $\Theta\left(\mathscr{S}_{1}, \mathscr{S}_{2}\right)$ if and only if for any $J \subset I$ either $\bigcup_{i \in J} U_{i} \in \mathscr{S}_{1}$ or $\bigcup_{i \in I \backslash J} V_{i} \in \mathscr{S}_{2}$ (Lemma 1). The proof of the fact that $\Theta\left(\mathscr{S}_{1}, \mathscr{S}_{2}\right)$ is a $Q$-system depends on the Tikhonov theorem (see the proof of Lemma 2), while for $\boldsymbol{\Theta}^{\prime}$ this is straightforward.

In general $\Theta \neq \Theta^{\prime}$. For example, let $X$ be a finite discrete space consisiting of more than one point, and let $Y=X$. If $\mathscr{S}_{1}$ is the family of all non-empty subsets of $X$ and $\mathscr{S}_{2}=\{X\}$, then $\mathscr{S}_{1}, \mathscr{S}_{2} \in \mathfrak{Q}(X)$ and the diagonal of $X \times X$ is in $\Theta\left(\mathscr{S}_{1}, \mathscr{S}_{2}\right)$ but not in $\boldsymbol{\Theta}^{\prime}\left(\mathscr{S}_{1}, \mathscr{S}_{2}\right)$.

3. The first version of this paper did not contain Theorem 3, and the proof of Theorem 2 was much more complicated. The manuscript was carefully read by Ernest Michael, who made many valuable suggestions, among them the following. If $\boldsymbol{\Theta}_{1}$ and $\boldsymbol{\Theta}_{2}$ are as in Theorem 2, let us say that $\boldsymbol{\Theta}_{2}$ is bigger than $\boldsymbol{\theta}_{1}$ if $\Theta_{1}\left(\left\{\mathscr{S}_{\alpha}\right\}\right) \subset \Theta_{2}\left(\left\{\mathscr{S}_{\alpha}\right\}\right)$ for any $\left\{\mathscr{S}_{\alpha}\right\} \in \prod_{\alpha} \mathfrak{Q}\left(X_{\alpha}\right)$. Michael noted that, since there exists a map $\Theta$ satisfying the requirements of Theorem 2 , there exists a biggest such $\Theta$ : just take the least upper bound of all such $\Theta$ 's. He asked if this biggest $\Theta$ can be described explicitly. Theorem 3 arose from an attempt to answer this question. It turns out that the $\Theta$ of Theorem 3 is just the biggest $\Theta$ :

Proposition 2. Let $\Theta_{M}: \prod_{\alpha \in A} \mathfrak{Q}\left(X_{\alpha}\right) \rightarrow \mathfrak{Q}\left(\prod_{\alpha \in A} X_{\alpha}\right)$ be the map described in Theorem 3, and let $\Theta: \prod_{\alpha \in A} \mathfrak{Q}\left(X_{\alpha}\right) \rightarrow \mathfrak{Q}\left(\prod_{\alpha \in A} X_{\alpha}\right)$ be any other continuous support-preserving map. Then $\boldsymbol{\Theta}_{M}$ is bigger than $\boldsymbol{\Theta}$.

Proof. Let $\left\{\mathscr{S}_{\alpha}\right\} \in \prod_{\alpha} \mathfrak{Q}\left(X_{\alpha}\right)$. To prove that $\boldsymbol{\Theta}\left(\left\{\mathscr{S}_{\alpha}\right\}\right) \subset \boldsymbol{\Theta}_{M}\left(\left\{\mathscr{S}_{\alpha}\right\}\right)$, we must show that, if $F=\prod_{\alpha} F_{\alpha}$ is an $\left\{\mathscr{S}_{\alpha}\right\}$-big box in $\prod_{\alpha} X_{\alpha}$, then every element of $\Theta\left(\left\{\mathscr{S}_{\alpha}\right\}\right)$ meets $F$. 
Let $\mathscr{S}_{\alpha}^{\prime}$ be the family of all open sets in $X_{\alpha}$ which meet $F_{\alpha}$. Then $\mathscr{S}_{\alpha} \subset$ $\mathscr{S}_{\alpha}^{\prime}$, so $\mathscr{S}_{\alpha}^{\prime}$ belongs to every neighborhood of $\mathscr{S}_{\alpha}$ in $\mathfrak{Q}\left(X_{\alpha}\right)$, and hence $\left\{\mathscr{S}_{\alpha}^{\prime}\right\}$ is in every neighborhood of $\left\{\mathscr{S}_{\alpha}\right\}$ in $\prod_{\alpha} \mathfrak{Q}\left(X_{\alpha}\right)$. Since $\boldsymbol{\Theta}$ is continuous, it follows that $\boldsymbol{\Theta}\left(\left\{\mathscr{S}_{\alpha}^{\prime}\right\}\right)$ is in every neighborhood of $\Theta\left(\left\{\mathscr{S}_{\alpha}\right\}\right)$ in $\mathfrak{Q}\left(\prod_{\alpha} X_{\alpha}\right)$, and therefore

$$
\Theta\left(\left\{\mathscr{S}_{\alpha}^{\prime}\right\}\right) \supset \Theta\left(\left\{\mathscr{S}_{\alpha}\right\}\right)
$$

Since $\mathscr{S}_{\alpha}^{\prime}$ is supported by $F_{\alpha}$ and $\Theta$ is support-preserving, $\Theta\left(\left\{\mathscr{S}_{\alpha}^{\prime}\right\}\right)$ is supported by $F$. Hence $F$ meets every element of $\Theta\left(\left\{\mathscr{S}_{\alpha}^{\prime}\right\}\right)$ and therefore every element of $\boldsymbol{\Theta}\left(\left\{\mathscr{S}_{\alpha}\right\}\right)$.

4. E. Michael observed that the theorem stating that perfect maps are preserved by arbitrary products follows from Theorem 3 . The argument is similar to the one used to deduce Theorem 1 from Theorem 2. Note that an onto map $f: X \rightarrow Y$ is perfect if and only if for every $y \in Y$ the system $\mathscr{S}_{y}$ of all open sets $V$ in $X$ with $f^{-1}(y) \subset V$ is a $Q$-system and the map $y \mapsto \mathscr{S}_{y}$ from $Y$ to $\mathfrak{Q}(X)$ is continuous.

5. Let $f_{\alpha}: X_{\alpha} \rightarrow Y_{\alpha}, \alpha \in A$, be a family of tri-quotient maps. For every $\alpha \in A$ there is a $t$-assignment $U \mapsto U^{*}\left(U \in \mathscr{T}\left(X_{\alpha}\right), U^{*} \in \mathscr{T}\left(Y_{\alpha}\right)\right)$ for $f_{\alpha}$. Combining Proposition 1, Theorem 3 and the argument after Theorem 2, we get a $t$-assignment $U \mapsto U^{*}\left(U \in \mathscr{T}\left(\prod_{\alpha} X_{\alpha}\right), U^{*} \in \mathscr{T}\left(\prod_{\alpha} Y_{\alpha}\right)\right)$ for $\prod_{\alpha} f_{\alpha}$ which can be described as follows.

For every $\alpha \in A$ let $p_{\alpha}: \prod_{\alpha} X_{\alpha} \rightarrow X_{\alpha}$ denote the projection. Let $U \in$ $\mathscr{T}\left(\prod_{\alpha} X_{\alpha}\right)$ and $\left\{y_{\alpha}\right\} \in \prod_{\alpha} Y_{\alpha}$. Then $\left\{y_{\alpha}\right\} \in U^{*}$ if and only if for every $\left\{V_{\alpha}\right\} \in \prod_{\alpha} \mathscr{T}\left(X_{\alpha}\right)$ such that $U \subset \bigcup_{\alpha \in A} p_{\alpha}^{-1}\left(V_{\alpha}\right)$ there exists $\alpha \in A$ such that $y_{\alpha} \in V_{\alpha}^{*}$.

\section{ACKNOWLEDGMENT}

The author is most thankful to Ernest Michael for many helpful discussions and suggestions. The present version of the paper appeared after Michael's valuable comments on earlier versions. Thanks are due also to the referee. The paper was prepared while the author was a guest of the Mathematical Institute of the Ludwig-Maximilian University in Munich, supported by an Alexander von Humboldt Research Fellowship. The author cordially thanks the Mathematical Institute in Munich and personally Professor W. Roelcke for their hospitality and the Humboldt Foundation for its support.

\section{REFERENCES}

[CČN] J. Chaber, M. Čoban, and K. Nagami, On monotonic generalizations of Moore spaces, Čech complete spaces and p-spaces, Fund. Math. 84 (1974), 107-119.

[E] R. Engelking, General topology, Heldermann Verlag, Berlin, 1989.

[H] O. Hájek, Notes on quotient maps, Comment. Math. Univ. Carolin. 7 (1966), 319-323.

[JW] W. Just and H. Wicke, Some conditions under which tri-quotient or compact-covering maps are inductively perfect, Topology Appl. 55 (1994), 289-305.

[M1] E. Michael, Bi-quotient maps and cartesian products of quotient maps, Ann. Inst. Fourier (Grenoble) 18 (1968), 287-302.

[M2] _ Complete spaces and tri-quotient maps, Illinois J. Math. 21 (1977), 716-733.

[M3] _ A note on bi-quotient and tri-quotient maps, Bull. Polish Acad. Sci. Math. 41 (1993), 285-291. 
[O] A. V. Ostrovsky, Triquotient and inductively perfect maps, Topology Appl. 23 (1986), 25-28.

[P] B. Pasynkov, Open mappings, Dokl. Akad. Nauk SSSR 175 (1967), 292-295; English transl., Soviet Math. Dokl. 8 (1967), 853-856.

Mathematisches Institut Der Ludwig-Maximilians-Universität, Theresienstrasse 39, D-80333 MÜNCHEN, GERMANY

Current address: Department of Mathematics, International Moscow University, Leningradskij prospekt 17, Moscow 125040, Russia 